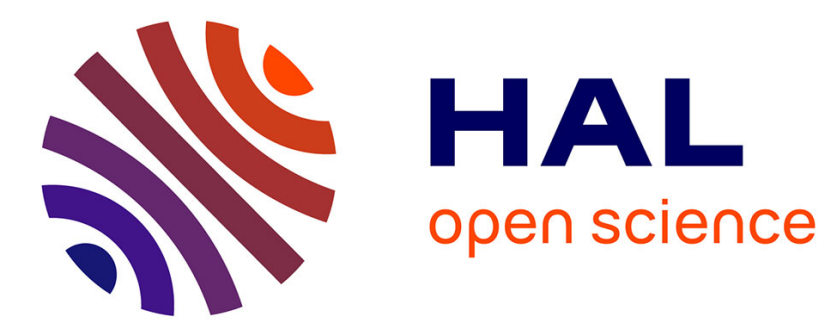

\title{
Conformation of branched polymers
}

\author{
M. Daoud, J.-F. Joanny
}

\section{To cite this version:}

M. Daoud, J.-F. Joanny. Conformation of branched polymers. Journal de Physique, 1981, 42 (10), pp.1359-1371. 10.1051/jphys:0198100420100135900 . jpa-00209328

\section{HAL Id: jpa-00209328 https://hal.science/jpa-00209328}

Submitted on 1 Jan 1981

HAL is a multi-disciplinary open access archive for the deposit and dissemination of scientific research documents, whether they are published or not. The documents may come from teaching and research institutions in France or abroad, or from public or private research centers.
L'archive ouverte pluridisciplinaire HAL, est destinée au dépôt et à la diffusion de documents scientifiques de niveau recherche, publiés ou non, émanant des établissements d'enseignement et de recherche français ou étrangers, des laboratoires publics ou privés. 


\title{
LE JOURNAL DE PHYSIQUE
}

J. Physique 42 (1981) 1359-1371

OCTOBRE 1981, PAGE 1359

Classification

Physics Abstracts

$05.50-61.40$

\section{Conformation of branched polymers}

\author{
M. Daoud and J. F. Joanny (*) \\ Laboratoire Léon Brillouin, CEN Saclay, B.P. no 2, 91190 Gif sur Yvette, France \\ $\left({ }^{*}\right)$ Physique de la Matière Condensée $\left({ }^{* *}\right)$, Collège de France, 11, place Marcelin Berthelot, 75231 Paris Cedex 05, France
}

(Reçu le 27 avril 1981, accepté le 25 juin 1981)

\begin{abstract}
Résumé. - Nous étudions la conformation de polymères branchés monodisperses en solutions diluée et concentrée.

Nous commençons par généraliser la théorie de champ moyen de Zimm et Stockmayer à l'étude de l'élasticité des chaînes et des corrélations de concentration à l'intérieur des chaînes. Cette théorie de champ moyen nous sert de point de départ pour construire une théorie de Flory des polymères branchés.

En solution diluée, en bon solvant nous retrouvons les résultats d'Isaacson et Lubensky. En solvant théta la théorie de champ moyen ne donne de résultats acceptables que pour des dimensions d'espace, $d$, supérieures à 6 . L'exposant de la variation du rayon de giration en fonction de la masse moléculaire, $R \sim N^{v}$, est $v=7 / 4(d+1)$.

En phase fondue les chaînes ne se recouvrent pratiquement pas et sont effondrées sur elles-mêmes, ce qui conduit à un exposant $v=1 / d$ pour le rayon de giration.

Dans tous ces régimes, nous donnons les lois d'échelle pour les propriétés locales des chaînes et nous étudions le passage vers les propriétés correspondantes des chaînes linéaires lorsque la fraction de branchement est réduite.
\end{abstract}

\begin{abstract}
We study the conformation of randomly branched, monodispersed polymers both in dilute and concentrated solutions in their reaction bath.

We first extend the Zimm-Stockmayer mean field theory to such properties as elasticity and concentration correlations. We then take this mean field theory as a basis for a Flory approach.

In a dilute solution, we recover previous results by Isaacson and Lubensky in a good solvent. In a theta solvent, the critical dimension above which the mean field approach is valid is 6 . The exponent for the molecular weight dependence of the radius of gyration, $R \sim N^{v}$, is $v=7 / 4(d+1)$, where $d$ is the dimension of space.

In a melt the polymer chains overlap very weakly, leading to an exponent $v=1 / d$.

In all these regimes, we give scaling laws for the local properties of the chain, and we study the cross-over to linear chain behaviour when the branching fraction is reduced.
\end{abstract}

1. Introduction. - Linear polymers are made of a succession of $N$ bifunctional monomeric units. Consider now a succession of tri (or multi) functional units, branched in a completely random way. The resulting species is a randomly branched polymer.

There has been recently a renewed interest in the conformational properties of such branched chains [1-5]. In their pioneering work, Zimm and Stockmayer $[6,7,18]$ considered the case when no excluded volume is present, so that two monomers can be on the same site. Their result is that the radius of a chain

(**) Equipe de recherche associée au C.N.R.S., ERA 542. is proportional to $N^{1 / 4} a, N$ being the number of statistical units and $a$ the step length. Introduction of the excluded volume effects leads to a swelling of the molecule. As a result, the radius behaves like $N^{v} a$, where the exponent $v$ has to be at least equal to $d^{-1}$ ( $d$ being the dimension of space) for large polymerization indices.

These branched polymers have recently been related to the "lattice animals" which appear in the percolation problem $[4,8,9]$. Thus the exponent $v$ is related to critical exponents. We expect it to become a constant, identical to the Zimm-Stockmayer value for large dimensions of space. Below a critical value $d_{\mathrm{c}}$ of the 
space dimension $d, v$ depends on $d$. It can be calculated in an $\varepsilon$ expansion $\left(\varepsilon=d_{\mathrm{c}}-d\right)$ using renormalization group techniques [4].

In this paper, we would like to study the statistics of monodisperse randomly branched polymers in their reaction bath both in the dilute and the concentrated limits, emphasizing on the radius of gyration and the pair correlation function inside the chains. This study is essentially based on a Flory type approach $[10,11]$. Using this method, de Gennes [3] has evaluated the critical dimensions $d_{\mathrm{c}}$ for a dilute solution in a good solvent, for a monodisperse melt, and for the gelation problem which corresponds to a polydisperse concentrated solution with a very specific molecular weight distribution. Isaacson and Lubensky [2] also used the same Flory arguments to calculate the exponent $v$ of the radius of gyration both in a good solvent and a monodisperse melt. We recover their result for a dilute branched chain in a good solvent but our value for the melt is in disagreement with theirs.

On the experimental side it is not clear whether it is actually possible to get completely randomly branched polymers. (We will specify later on what we mean by completely random.) However one may reasonably assume that in certain cases the branching is sufficiently random [7] and that the branched polymers behave as randomly branched polymers. The other experimental problem which always occurs in the process of forming branched polymers is the polydispersity of the samples. But there are now powerful techniques available to get rid of the polydispersity and operate with a rather narrow molecular weight distribution. Finally, we will also study the cross-over from branched to linear chain behaviours when branching is reduced.

In section 2 we will come back on the ZimmStockmayer mean field theory calculating the elastic free energy of a single chain and looking at the correlations inside a chain. Section 3 deals with the statistics of dilute chains both in a good and in a theta solvent. The last section is devoted to the study of monodisperse melts.

2. The Zimm-Stockmayer mean field theory. In this section we rederive the main results of the Zimm-Stockmayer $[6,18]$ theory, which is the mean field theory for the branched polymer problem. In this model, we build a branched tree (no loops allowed) from a solution of bifunctional and trifunctional units. The total number of units is $N$. During the chemical process, the activity $\Lambda^{2}$ of the trifunctional units is held constant. In this model, and in all the following, by randomly branched we will always mean branched at a fixed activity for the multifunctional units.

The Zimm-Stockmayer theory makes two basic assumptions :
- It assumes that the randomly branched polymer does not contain any loop. Thus we will consider only randomly branched trees. This means for instance that if we choose two monomers $i$ and $j$, in a given chemical configuration there is only one path along the chemical sequence connecting $i$ and $j$.

- It neglects all the excluded volume effects. As we will see later, this approximation does not describe the actual statistics of a branched polymer neither in a theta solvent nor in the melt as it does for linear chains.

It has to be noted at this point that excluded volume effect and loop formation are not two independent effects : if we consider the branching of the polymers in their reaction bath, the chemical configuration (the number of loops, of end points, ...) does depend on the interaction between solvent and monomeric molecules.

In the framework of this mean field theory Zimm and Stockmayer were the first to calculate the average radius of gyration of randomly branched polymers. Their result, rederived in appendix A, reads

$$
\begin{aligned}
& \left\langle R_{\mathrm{G}}^{2}\right\rangle=\frac{\pi^{1 / 2}}{4}\left(\frac{N}{\Lambda}\right)^{1 / 2} a^{2} \quad(\Lambda N \gg 1) \\
& \left\langle R_{\mathrm{G}}^{2}\right\rangle=\frac{N}{6}\left(1-\frac{\Lambda^{2} N^{2}}{30}\right) a^{2} \quad(\Lambda N \ll 1) .
\end{aligned}
$$

In order to provide a better understanding (hopefully) for the $\Lambda$ dependence in the highly branched case, we might give a blob-type [11, 14] argument.

If we have only trifunctional units, the average radius of gyration scales like $N^{1 / 4}$. When $\Lambda$ is smaller than unity the average number of monomers between trifunctional units is $\left(^{1}\right) n \sim \Lambda^{-1}$. These monomers form a gaussian chain with size $\xi \sim n^{1 / 2} a$. If we group the monomers in blobs, each of them made of $n$ units, we can represent the branched chain as a chain made exclusively of trifunctional units. In the process, the step length has become the blob with size $\xi$ (see Fig. 1). The radius of gyration is then :

$$
R_{\mathrm{G}}=\left(\frac{N}{n}\right)^{1 / 4} \xi \sim\left(\frac{N}{\Lambda}\right)^{1 / 4} a .
$$

When $\Lambda$ becomes of the order of $N^{-1}$, there is only one blob, and there is a smooth cross-over from the highly branched behaviour relation (1a) to the behaviour of an ideal linear gaussian chain, equation (1b). We are now interested in two other physical properties of branched chains :

i) The concentration correlation function.

ii) The elastic modulus.

a) Correlation function $(\Lambda N \gg 1)$. - The correlation function between monomers inside a chain, is closely related to the intensity measured in a light or neutron

( $\left.{ }^{1}\right)$ See appendix A. 


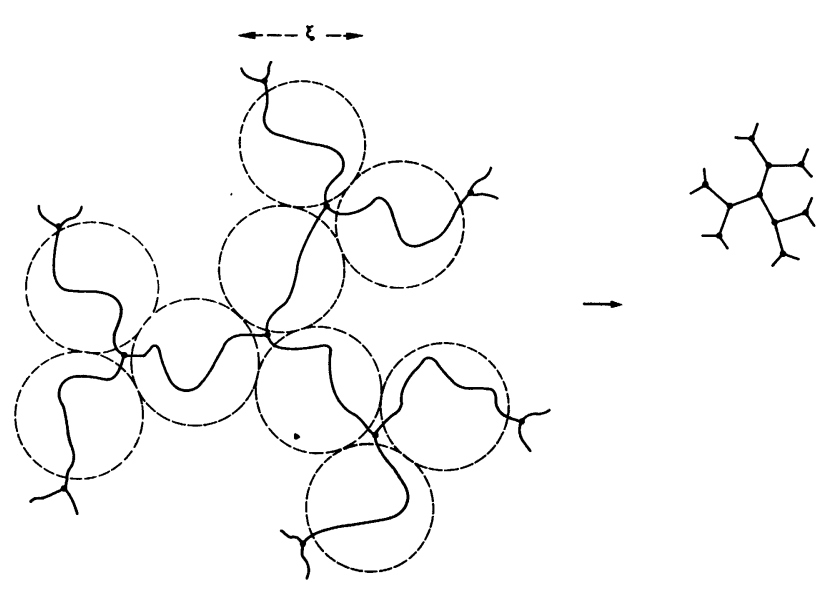

Fig. 1. - Schematic of the blob representation for a single branched chain : we group the monomers in blobs, with size $\xi$, made of $n \sim \Lambda^{-1}$ elements joined in a linear sequence. The molecule is then a branched ensemble of blobs.

scattering experiment. Let us call $C g(r)=\langle C(0) C(r)\rangle$ and $S(q)$ its Fourier transform.

- For very low $q\left(q R_{\mathbf{G}} \ll 1\right)$, we are in the Guinier range

$$
S(q)=N\left(1-q^{2} \frac{R_{\mathrm{G}}^{2}}{3}\right) .
$$

- For very large $q$, as scattering experiment probes length scales of size $q^{-1}$, it will probe the configuration of a blob, which is a gaussian chain as long as $q \xi \gg 1$, so that

$$
S(q) \sim \frac{1}{q^{2} a^{2}} \quad\left(q \gg \xi^{-1}\right) .
$$

- In the intermediate range, $R_{\mathrm{G}}^{-1}<q<\xi^{-1}, S(q)$ may be conjectured by a scaling argument : $S(q)$ is the number of monomers correlated in a region of size $q^{-1}$. Assuming (10a) for the radius of this region, we have

leading to

$$
q^{-1} \sim\left[\frac{S(q)}{\Lambda}\right]^{1 / 4} a
$$

$$
S(q) \sim \frac{\Lambda}{(q a)^{4}} \quad\left(R_{G}^{-1}<q<\xi^{-1}\right) .
$$

One can easily check that this last relation crosses over smoothly to equations (1) and (2) respectively for $q \sim R_{\mathrm{G}}^{-1}$ or $q \sim \xi^{-1}$.

b) Elasticity of randomly branched trees. - In order to define an elastic modulus (or an elastic free energy), one has to be very careful. There are actually several ways of defining an elasticity leading to different answers for the elastic modulus. The first way we can think of is to pull on two given end points of the polymer. In this process, what one measures is the elasticity of the linear chain joining these two end points. As we neglect the interactions, the « side chain » are not affected by the elongation and the elastic energy is

$$
\frac{F}{T}=\frac{\delta R^{2}}{P}
$$

$\delta R$ being the elongation and $P$ the average number of monomers between the two end points.

A much more interesting way to define an elasticity - which will be useful in the swelling of the chain - is to define a process involving all the monomers of the molecule. Such an elasticity may also be used in the study of such properties as excluded volume effects within the framework of a mean field theory, and of chain conformation in certain velocity gradients.

The simplest way to define such an elasticity seems to put all the monomers in a harmonic potential

$$
V=T \frac{\gamma}{N}\left(\sum_{i} S_{i}^{2}\right)=T \gamma R_{\mathrm{G}}^{2}
$$

where $S_{i}$ is the distance from monomer $i$ to the centre of mass of the chain. In this section we calculate the free energy of a chain in such a potential.

The partition function of the chain in the external potential $V$ is

$$
Z=\sum_{\text {config. }} \mathrm{e}^{-V / T}=\sum \mathrm{e}^{-i R_{G}^{2}}
$$

expanding (14) in powers of $\gamma$ :

$$
Z=Z_{0}\left\{1-\gamma\left\langle R_{\mathrm{G}}^{2}\right\rangle+\frac{\gamma^{2}}{2}\left\langle R_{\mathrm{G}}^{4}\right\rangle+\cdots\right\}
$$

where $Z_{0}$ is the partition function of the chain without any constraint $(\gamma=0)$. The free energy is then calculated to be

$$
F=F_{0}+T \gamma\left\langle R_{\mathrm{G}}^{2}\right\rangle \text {. }
$$

The variation of the radius of gyration $\delta R^{2}$ is

$$
\begin{aligned}
\left\langle\delta R^{2}\right\rangle=-\frac{\partial \log Z}{\partial \gamma}-\left\langle R_{\mathrm{G}}^{2}\right\rangle= & \\
& =\gamma\left[\left\langle R_{\mathrm{G}}^{4}\right\rangle-\left\langle R_{\mathrm{G}}^{2}\right\rangle^{2}\right] .
\end{aligned}
$$

Thus the variation in the elastic free energy due to a variation $\delta R^{2}$ of the radius of gyration is

$$
\frac{\delta F}{T}=\frac{\delta R^{2}\left\langle R_{G}^{2}\right\rangle}{\left\langle R_{G}^{4}\right\rangle-\left\langle R_{G}^{2}\right\rangle^{2}} .
$$

It is interesting to note that for ideal linear gaussian chains, $\left\langle R_{\mathrm{G}}^{4}\right\rangle$ is proportional to $\left\langle R_{\mathrm{G}}^{2}\right\rangle^{2}$. (The reason is that calculating all the averages, one just has to use a gaussian distribution [10].)

A second point we may look at qualitatively on relation (8) is the influence of loops. If the number of loops in the polymer is important, the fluctuations of $R_{\mathrm{G}}^{2}$ become smaller and the free energy change is 
increased. This is consistent with what one would expect : more cross-linking increases the elastic modulus.

In appendix B, we make an explicit calculation of the elastic free energy (8) :

$$
\frac{F}{T}=\frac{135}{2} \sqrt{\pi} \frac{\delta R^{2}}{\left(\frac{N}{\Lambda}\right)^{1 / 2} a}=\frac{135 \pi}{8} \frac{\delta R^{2}}{\left\langle R_{\mathrm{G}}^{2}\right\rangle}
$$

This calculation thus confirms the conjecture by Isaacson and Lubensky about the structure of the elastic energy.

All the above results derived in this mean field theory (1), (4), (9) are however highly unphysical and cannot be valid for space dimensions $d$ lower than 4 . The radius of gyration of a polymer, for instance, has to be larger than the collapsed value $N^{1 / d} a$. So the exponent $v$ has to be larger than $d^{-1}$. The mean field value $v=1 / 4$ is thus unacceptable for any physical $(d=3)$ situation, even in the melt or at the theta temperature, as was the case for linear polymers (where the mean field value is $v=1 / 2$ ).

Another way to realize how unphysical these results are is to look at the correlation function $g(r)$. In the intermediate regime $\xi<r<R_{G}, g(r)$ is the Fourier transform of $\Lambda / q^{4}$, so $g(r) \sim r^{4-d}$ which would mean that for $d<4 g(r)$ is an increasing function of the distance $r$.

However, although this study is unphysical for low space dimensions, it is far from being useless : as we will see, it describes the behaviour of branched polymers at higher dimensions, and it will be used as the starting point for a Flory-type theory in the following sections.

3. Dilute solutions. - In this section, we consider a randomly branched polymer in the high branching limit $(\Lambda N \gg 1)$ in a very dilute solution. We are going to study successively the situations in which the binary interactions are positive (good solvent) and vanish (theta solvent).

3.1 GoOD SOLVENT LIMIT. - a) Radius of gyration. - The free energy of a polymer chain in a solvent is the sum of its elastic free energy $F_{\mathrm{el}}$ and an interaction term $F_{\text {int }}$. In the usual Flory approximation, the elastic contribution is simply taken as its mean field evaluation. The interaction term is expanded in powers of the concentration inside the coil :

$$
F_{\text {int }}=v \frac{N^{2}}{R^{d}}+w \frac{N^{3}}{R^{2 d}}+\cdots+t_{n} \frac{N^{n}}{R^{(n-1) d}}+\cdots
$$

where $v$ is the excluded volume parameter and depends on temperature. It vanishes at the theta temperature.
All the other coefficients $(w, \ldots)$ are supposed to be positive constants.

In a good solvent, $v$ is positive and we can neglect the higher order interaction terms $\left({ }^{2}\right)$. The free energy of the chain is, dropping all the numerical constants

$$
\frac{F}{T}=\frac{R^{2}}{R_{\mathrm{G}}^{02}}+v \frac{N^{2}}{R^{d}}
$$

$R_{\mathrm{G}}^{0}$ being the ideal radius, relation $(10 a)$.

Minimization with respect to $R$ leads to the equilibrium radius or gyration

$$
\begin{gathered}
R_{\mathrm{G}} \sim N^{v} \Lambda^{-x} v^{1 /(d+2)} \\
v=\frac{5}{2(d+2)} \\
x=\frac{1}{2(d+2)} .
\end{gathered}
$$

The exponent $v$ has already been derived by Isaacson and Lubensky [2]. It depends on space dimension and goes back to the Zimm-Stockmayer value for a space dimension $d=8$. This critical dimension $d_{\mathrm{c}}=8$ has already been suggested by de Gennes [3]. For $d=3$, the exponent $v$ is equal to the exact solution to the problem recently given by Sourlas and Parisi [17]. A deeper understanding of this result, equation (12), can be given by a blob analysis : let us again group the monomers in subunits of $n$ monomers $\left(n \sim \Lambda^{-1}\right.$ being the average number of bifunctional monomers between trifunctional units). We call blobs these subunits. In a good solvent, such blobs are swollen and the radius $\xi$ of a blob is

$$
\xi \sim n^{3 /(d+2)} a \quad(d<4) .
$$

Considering the polymer as a randomly branched chain of 3 functional blobs with elementary length $\xi$ we get a radius

$R \sim\left(\frac{N}{n}\right)^{5 / 2(d+2)} \xi \sim N^{5 / 2(d+2)} \Lambda^{-1 / 2(d+2)} a(d<4)$.

Note that for dimensions between 4 and 8 , the blobs consisting of linear chains are not swollen any more $[11$, 10], and

$$
\xi \sim n^{1 / 2} a
$$

leading to a radius of gyration

$$
\begin{aligned}
R \sim\left(\frac{N}{n}\right)^{5 / 2(d+2)} \xi \sim N^{5 / 2(d+2)} & \Lambda^{(3-d) / 2(d+2)} a \\
& (4<d<8) .
\end{aligned}
$$

( ${ }^{2}$ ) It can be checked at the end of the calculation that this is legitimate at least for space dimensions $d>\overline{4 / 3}$. 
Note that this last expression (14) for the radius cannot be obtained from (24) : for $d>4$, we know that there is no swelling of the linear parts of the molecule, whereas (11) does not take this into account and deals with linear and branched parts on an equal footing. One can check easily that equation (14) crosses over smoothly to (13) and to the ZimmStockmayer [6] expression (1) respectively for $d=4$ and $d=8$. Also, it can be checked that we recover the linear chain behaviour

$$
\begin{array}{ll}
R \sim N^{3 /(d+2)} a & (d<4) \\
R \sim N^{1 / 2} a & (d>4)
\end{array}
$$

when the branching is decreased, the cross-over occurring for $\Lambda N \sim 1$.

b) Correlation function. - The blob analysis suggested above leads us naturally to a scaled form for the pair correlation function $S(q)$.

- For very low values of $q\left(q R_{\mathrm{G}} \ll 1\right)$ in the Guinier range

$$
S(q)=N\left(1-\frac{q^{2} R_{\mathrm{G}}^{2}}{3}\right)
$$

- For very large values of $q(q \xi \gg 1), S(q)$ probes the interior of the blobs, which are part of linear chains. It has been shown that we have then

$$
\begin{aligned}
& S(q) \sim \frac{1}{q^{(d+2) / 3}} \quad d<4 \\
& S(q) \sim \frac{1}{q^{2}} \quad d>4
\end{aligned}
$$

In the intermediate range $\left(R^{-1}<q<\xi^{-1}\right), S(q)$ may be obtained by scaling arguments. We get

$$
S(q) \sim \frac{\Lambda^{1 / 5}}{q^{2 / 5(d+2)}} \quad(d<4) .
$$

A qualitative plot of $S(q)$ is shown on figure 3 for space dimension $d=3$.

c) Osmotic pressure virial coefficient. - In a dilute good solvent solution, the coils made by branched polymers show a strong repulsive interaction and are more concentrated than linear chains of the same molecular weight. It is thus legitimate to assume that they behave as hard spheres. This leads to a second virial coefficient $A_{2}$ between monomers

$$
A_{2} \sim T \frac{R^{d}}{N^{2}}
$$

For usual three dimensional systems, $A_{2}$ scales like $N^{1 / 2}$, and the osmotic pressure for a dilute solution of branched polymers is of the form

$$
\frac{\pi}{T}=\frac{C}{N}+\frac{C^{2}}{N^{1 / 2}}+\cdots
$$

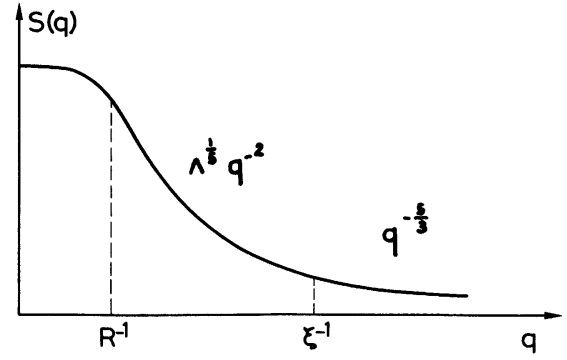

Fig. 3. - Schematic plot of the scattered intensity $S(q)$ by a single chain : for $d=3$. One can see 3 different regions : - in the Guinier range $(q R \ll 1) S(q) \sim N\left(1-\frac{q^{2} R^{2}}{3}\right)$ may provide a measure of the radius of gyration $R$; - in the intermediate range $\left(R^{-1}<q<\xi^{-1}\right)$ the experiment probes the local branched nature of the chain : $S(q) \sim \Lambda^{1 / 5} q^{-2}$; - for higher values of $q\left(\xi^{-1}<q<a^{-1}\right)$, the linear blob behaviour is seen : $S(q) \sim q^{-5 / 3}$. All these regimes are within a Neutron Small Angle Scattering range.

d) Importance of the loops. - In the preceding sections, we considered the effect of the repulsive binary interactions without talking about loops. In fact, it turns out that if we build a randomly branched polymer starting from a solution of bi- and trifunctional units in a good solvent, both numerical simulations [15] and field theory techniques [4] show that on the average the number of loops formed is a non critical quantity. In other terms, this number remains finite when the molecular weight of the polymer goes to infinity. Thus in this case it seems to be legitimate to neglect loops.

However, if we do not study the polymer in its reaction bath, the situation may be very different : At the theta temperature $(v=0)$ or in a poorer solvent $(v<0)$ the chain is more compact and the number of loops may become much more important. If we then put the polymer thus formed back in a good solvent, the statistics may become very different. We do not consider this case here.

3.2 Theta solvent. - In most practical cases, the excluded parameter $v$ is temperature dependent and vanishes at a temperature which we call $\Theta$, the Flory compensation temperature. At this temperature, the above results (Eq. (12) and below) break down, and we have to go back to the expression (10) for the free energy :

$$
F=\frac{R^{2}}{R_{\mathrm{G}}^{02}}+w \frac{N^{3}}{R^{2 d}}\left({ }^{3}\right) .
$$

Then we may first look at the critical dimension above which the Zimm-Stockmayer result holds. This happens if the last term on the right hand side of (18),

$\left({ }^{3}\right)$ We drop all the higher order terms, and it can be checked at the end of the calculation that they are negligible at least for space dimensions $d>4 / 3$. 
calculated for the Zimm-Stockmayer value of the radius is much less than unity.

We find

$$
\begin{gathered}
3-\frac{d}{2}<0 \\
d_{\mathrm{c}}^{\Theta}=6 .
\end{gathered}
$$

A result which is consistent with the field theoretical evaluation of Isaacson and Lubensky [2].

For lower space dimensions, the equilibrium radius of gyration is found by minimizing (18) with respect to $R$ :

$$
\begin{gathered}
R \sim N^{v_{\theta}} a \\
v_{\theta}=\frac{7}{4(1+d)} .
\end{gathered}
$$

It has to be noted that for instance for $d=3$, the mean field theory does not apply. For $d=3$, the radius of a chain in a theta solvent is close to the radius in a good solvent $\left(v_{\theta}=7 / 16\right.$ versus $\left.v=1 / 2\right)$ which means that in such systems the three body interaction is almost as important as the two body interaction.

A blob analysis may give a description of the behaviour of the chain : let us again group the monomers in subunits of $n$ monomers, which we call blobs $\left(n \sim \Lambda^{-1}\right.$ is still the average number of bifunctional units between trifunctional units). In a theta solvent, these blobs are roughly ideal for $d \geqslant 3$, and the radius $\xi$ of a blob is

$$
\xi \sim n^{1 / 2} a \quad(d \geqslant 3) .
$$

Considering the polymer as a randomly branched chain of three-functional blobs with unit length $\xi$ we get a radius

$$
R \sim\left(\frac{N}{n}\right)^{v_{\theta}} \xi \sim N^{7 / \dot{4}(d+1)} \Lambda^{(5-2 d) / 4(d+1)} a .
$$

For space dimensions lower than 3 , the linear blob is swollen in the theta regime. Within a Flory theory, we have then

$$
\xi \sim n^{2 /(d+1)} a \quad(d<3)
$$

leading to

$$
R \sim N^{7 / 4(1+d)} \Lambda^{-1 / 4(d+1)} a \quad(d<3) .
$$

The reader may check easily that (20a) crosses over to the Zimm-Stockmayer result, equation ( $1 a)$, for $d=6$, and that one gets back to the linear chain behaviour when $\Lambda N \sim 1$ both from $(20 a)$ and $(20 b)$ respectively for $d>3$ and $d<3$.

An important point has to be noted here : in this discussion, we supposed that the excluded volume parameter $v$ is the same for bifunctional and trifunc- tional units. This means that we suppose that there is only one theta temperature. Experimentally, one may distinguish two different cases :

(i) systems for which the theta temperatures corresponding to pure bifunctional $\Theta_{2}$ and pure trifunctional units $\Theta_{3}$ are close to each other. One may define an effective $\Theta$ temperature for the mixture of bi and trifunctional units. Then the above results apply directly ;

(ii) systems for which $\Theta_{2}$ and $\Theta_{3}$ are very different from each other. In this case, one would have to make a somewhat more tedious blob analysis corresponding to the different possibilities that might be encountered. This will not be done here.

4. Concentrated solutions. - 4.1 MonODISPERSE MELT. - It has been recognized a long time ago by Flory [10] that melts of linear chains can be described by a simple mean field theory. In this section, we argue that the situation is not so simple for randomly branched chains. In linear melts, the important property is that the different chains do overlap (at least for $d \geqslant 2[11]$ ). It is argued here that in monodisperse melts, the different branched molecules do not overlap in actual experiments $(d<4)$.

For large space dimensions, we expect the ZimmStockmayer mean field theory to describe the statistics of a chain in the melt. As we said earlier, below $d=4$, this theory gives unphysical results. Thus the critical dimension $d_{\mathrm{c}}$ for the melt is greater than (or equal to) 4 . For large dimensions, the radius scales like $N^{1 / 4}$ and the chains do overlap.

In order to study randomly branched melts, let us first use a Flory-Huggins [10] type free energy. We focus on one chain (a labelled chain) and we call $\varphi$ the volume fraction of monomers belonging to this chain. The free energy of the chain reads

$$
\frac{F}{T}=\frac{R^{2}}{R_{\mathrm{G}}^{02}}+R^{d}\left[\frac{1-\varphi}{N} \log (1-\varphi)\right] .
$$

The first term is the elastic contribution derived in the first section. The second term is the translational energy of the other chains. This free energy is valid under the assumption that the chains do overlap.

Minimization of the free energy (21) gives a concentration $\varphi=1$ for dimensions lower than 4 and the usual mean field result for $d>4$. From this approach, it seems that for melts $d_{\mathrm{c}}=4$, and that below $d_{\mathrm{c}}$ the chains do not overlap and have thus a radius $R$ proportional to $N^{1 / d}$.

If we now suppose that the chains do not overlap, we can use the free energy (10). But we have to keep all the terms in the expansion, the higher order terms being dominant [13]. Minimization of such a free energy gives a radius

$$
R \sim N^{1 / d} a \quad(d<4)
$$

and a critical dimension $d_{\mathrm{c}}=4$. 
The equilibrium radius does not have a proper cross-over towards the linear chain radius in the limit $\Lambda N=1$. In fact, the concentration inside the chain cannot be one and fall down to zero discontinuously. At the edge of each coil, there is an interfacial layer inside which the concentration goes down. Inside this layer, there may be an overlap of the neighbouring chains. Inside this layer, we expect the chains to behave as linear chains, and when the thickness $\lambda$ of this layer becomes of the order of the radius, we should reach the linear chain limit $\Lambda N \sim 1$ (see section $2 . \mathrm{b}$ below).

In the same way, equation (22) fails to give back the Zimm-Stockmayer result, equation ( $1 a)$, for $d=4$ as expected. In fact, the weakness of Flory's approach as developed here - which has already been mentioned above - is that it deals with the tri and bifunctional units on an equal footing : in the case of melts, what is implicitly supposed is that everything is collapsed. We know that this is not true for the linear parts of the chain, at least for dimensions above 2. For lower dimensions $(d \leqslant 2)$, we know $[11,13]$ that the linear parts are also collapsed, and we thus expect equation (22) to be valid. For larger dimensions $(d>2)$, we are going to make a blob analysis in order to have an understanding of the conformation of the branched chain. As before, we group the monomers in blobs with radius $\xi$, made of $n$ elements each. $n \sim \Lambda^{-1}$ corresponds to the linear parts between trifunctional units. The radius $\xi$ of a blob is, because we are in the melt :

$$
\begin{array}{ll}
\xi \sim n^{1 / d} a & (d<2) \\
\xi \sim n^{1 / 2} a & (d>2) .
\end{array}
$$

The radius of the chain is then, for $d<4$

$$
R \sim\left(\frac{N}{n}\right)^{1 / d} \xi
$$

leading to

$$
\begin{array}{ll}
R \sim N^{1 / d} a & (d<2) \\
R \sim N^{1 / d} \Lambda^{(2-d) / 2 d} a & (2<d<4) .
\end{array}
$$

Note that for $d=4$, equation (22a) leads back to Zimm-Stockmayer's equation (1a).

Thus we define a blob length $\xi$

$$
\xi \sim \Lambda^{-1 / 2} a \quad(d>2)
$$

inside which the molecule has a linear chain behaviour.

An important point should be noted here concerning the overlap of the molecules : let us calculate the contribution to the concentration due to a molecule in a sphere of radius $R$

$$
\begin{gathered}
C_{\mathrm{s}} \sim \frac{N}{R^{d}} \sim \Lambda^{(d-2) / 2} a^{-d} \quad(2<d<4) \\
\sim \quad a^{-d} \quad(d<2) .
\end{gathered}
$$

This shows that in the melt, if we have only multifunctional units $(\Lambda \sim 1)$ there is no overlap of the different molecules. The polymer itself can build up the whole concentration. On the contrary when $\Lambda$ is smaller than unity, there is some overlap of the molecules in order to build up the total concentration. From relation (24), we find that in the sphere with radius $R$, the average number of different molecules is

$$
N_{\mathrm{b}} \sim \Lambda^{(2-d) / 2} \quad(2<d<4)
$$

and we come to the interesting result that the amount of overlap is controlled only by the activity $\Lambda$ of the multifunctional units. Finally, when $\Lambda$ is decreased, $N_{\mathrm{b}}$ crosses over to the linear chain value [11] $N^{1}$ when $\Lambda \sim N^{-1}$

$$
N^{1} \sim N^{(d-2) / 2} .
$$

Let us note that the degree of overlapping of the branched chains is much smaller than that of linear chains when the chain is highly branched $(\Lambda N \gg 1)$

$$
\frac{N_{\mathrm{b}}}{N^{1}} \sim(\Lambda N)^{(2-d) / 2} \ll 1 .
$$

Finally, comparing (22a) to (23) when $\Lambda N \sim 1$, we can see that there is a cross-over from the branched chain to the linear polymer behaviour. Note that in this limit, the overlap between the molecules is very high $\left(N_{\mathrm{b}} \sim N^{1} \sim N^{(d-2) / 2} .2<d<4\right)$.

This was not included in our Flory approach, which supposed that the molecules do not overlap. On the other hand, this approach is valid either when there is no bifunctional units $(\Lambda \sim 1)$ or when $d<2$, when the linear chains themselves do not overlap.

Another interesting question is to determine the correlation function inside a labelled chain. This function $S(q)$ is proportional to the intensity in a Neutron Scattering experiment where some chains have been labelled (by deuteration for instance).

- For very low $q(q R \ll 1)$ we still have

$$
S(q) \simeq N\left(1-\frac{q^{2} R^{2}}{3}\right)
$$

where $R$ is given by (22).

- For very large $q\left(a^{-1}<q<\xi^{-1}\right)$ the scattering experiment probes the interior of a blob, which is a linear chain

$$
S(q) \sim \frac{1}{q^{2} a^{2}} .
$$

- In the intermediate range $\left(R^{-1}<q<\xi^{-1}\right)$, the situation is less clear. In order to get the scattered intensity, one needs to have a precise idea about the concentration profile in the molecule : a constant value of the concentration dropping at the interface would lead to the characteristic $S(q) \sim q^{-4}$ scattering law, whereas a more gradual decrease, allowing more 
fluctuations would lead to $S(q) \sim q^{-d} \Lambda^{(d-2) / 2}$. As we do not know the precise concentration profile at the present time, we will not continue this discussion. It would certainly be very interesting to perform the corresponding Neutron Scattering experiments on a melt (or a semi dilute solution) where some chains are labelled by deuteration in the intermediate range. These experiments would make easily the difference between a $q^{-4}$ and a $q^{-3}$ scattering law, thus leading to a more precise idea about the concentration profile.

4.2 SEMI Dilute SOlutions. - Starting from a dilute solution in a good solvent, and increasing the concentration $C$, we come to a point where the different chains are in close contact with each other. This defines a concentration $C^{*}$, analogous to the so called overlap concentration for linear chains [11, 12, 14].

$$
C^{*} \sim \frac{N}{R^{d}} \sim N^{1-v d} .
$$

As for linear polymers, we call semi dilute solution of randomly branched chains a solution with a concentration $C>C^{*}$. In all the following, we are going to restrict our discussion on the case $d=3$ which is most interesting for experimental purpose.

a) The non overlapping regime. - We start from concentrations just above $C^{*}$ and increase the concentration. Because we are in a semi dilute solution, we are led to define two very different blobs.

(i) The linear blob, $\xi_{\Lambda}$, which is made of the linear part of the chains. This has already been defined in section (3.a); it is made of $g_{\Lambda} \sim \Lambda^{-1}$ elements, and we have

$$
\xi_{\Lambda} \sim \Lambda^{-3 / 5} a .
$$

(ii) In the same way as for linear chains, we may also define a concentration blob, made of $g_{c}$ elements with size $\xi_{\mathrm{c}}$. This is a branched blob corresponding to the local part of the branched molecule which behaves as a single chain. The radius $\xi_{\mathrm{c}}$ of this blob can be obtained by assuming a scaling law for the radius of the molecule

$$
\xi_{\mathrm{c}} \simeq R_{\mathrm{d}} f\left(\frac{C}{C^{*}}\right)
$$

where $R_{\mathrm{d}}$ is the radius of a single chain, equation (12) :

$$
R_{\mathrm{d}} \sim N^{1 / 2} \Lambda^{-1 / 10} a
$$

and $f(x)$ is a universal function.

Combining (25) with the last two equations gives

$$
\xi_{\mathrm{c}} \sim N^{1 / 2} \Lambda^{-1 / 10} \text { af }\left(C a^{3} N^{1 / 2} \Lambda^{-3 / 10}\right) \text {. }
$$

Because the blob is a local part of the chain $\xi_{\mathrm{c}}$ does not depend on the total length $N$ of the molecule. Then supposing that $f(x)$ behaves as a power law, we get

$$
\xi_{c} \sim \Lambda^{1 / 5} C^{-1} a^{-2} .
$$

As we said above, this " branched blob ", made of $g_{\mathrm{c}}$ elements corresponds to the local part of the molecule which behaves like a single chain. Thus

$$
\xi_{\mathrm{c}} \sim\left(\frac{g_{\mathrm{c}}}{g_{\Lambda}}\right)^{1 / 2} \xi_{\Lambda}
$$

leading to

$$
g_{\mathrm{c}} \sim C^{-2} \Lambda^{3 / 5}
$$

Finally, the molecule is a condensed ensemble of concentration blobs :

$$
\begin{aligned}
& R \sim\left(\frac{N}{g_{\mathrm{c}}}\right)^{1 / 3} \xi_{\mathrm{c}} \\
& R \sim\left(\frac{N}{C}\right)^{1 / 3} .
\end{aligned}
$$

Relation (29) shows that in this regime, the chains do not overlap. By this, we mean that the concentration due to the molecule itself in the sphere of radius $R$ is sufficient to build up the total concentration. This situation is to be compared with the more concentrated regime in the next section. Of course, there is some overlap between the molecules in an interfacial layer, with size $\xi_{A}$ where linear dangling ends might overlap.

An important point in this regime concerns the size $\xi_{\Lambda}$ of the linear parts, which is smaller than the size of the branched concentration blob $\xi_{\mathrm{c}}$ (in other terms, $\left.g_{\Lambda} \ll g_{\mathrm{c}}\right)$.

When we increase the concentration, because the concentration blob size depends strongly on concentration, we reach a concentration $\tilde{C}$ when the linear blob and the concentration blob are of the same order :

$$
\begin{aligned}
\xi_{c}(\tilde{C}) & \sim \xi_{\Lambda} \\
\tilde{C} & \sim \Lambda^{4 / 5} .
\end{aligned}
$$

For concentrations below $\tilde{C}$, the linear chains are inside the concentration blob and do not overlap. This is reversed above $\widetilde{C}$.

It is interesting to notice that $\tilde{C}$ is exactly the overlap concentration for a solution of swollen chains made of $n \sim \Lambda^{-1}$ monomers.

b) The overlapping regime. - For concentrations $C$ above $\tilde{C}$, the single chain behaviour is within the linear parts. Then, we know from previous work on linear chains $[11,14]$ that for distances larger than $\xi_{c}$, the linear chains do overlap. The size $\xi_{\mathrm{c}}$ of the concentration blob is then the same as for a semi dilute solution of linear chains $[11,14]$ :

$$
\begin{aligned}
\xi_{c} & \sim\left(C a^{3}\right)^{-3 / 4} a \\
g_{c} & \sim\left(C a^{3}\right)^{-5 / 4} .
\end{aligned}
$$

In the same way, the « linear blob $»$, made of $g_{A} \sim \Lambda^{-1}$ 
elements with size $\xi_{A}$ is now roughly and ideal string of concentration blobs :

$$
\begin{aligned}
& \xi_{\Lambda} \sim\left(\frac{g_{A}}{g_{\mathrm{c}}}\right)^{1 / 2} \xi_{\mathrm{c}} \\
& \xi_{\Lambda} \sim \Lambda^{-1 / 2}\left(C a^{3}\right)^{-1 / 8} a
\end{aligned}
$$

let us emphasize the difference between the non overlapping and this regime :

We define a concentration blob, $\xi_{\mathrm{c}}$. This may be larger or smaller than the linear parts of the chain between trifunctional units :

- When it is larger, it has the same behaviour as the single branched chain considered in section (3). The chain is then a collapsed ensemble of these blobs and we find that there is basically no overlap between the different molecules, except in an interfacial layer.

- When it is smaller, the concentration blob is the same as for linear polymers. Then both the concentration and the linear blobs are derived from what we know about linear chains. Among other, there is an overlap of the molecules. Finally, the whole branched chain is still a collapsed ensemble of linear blobs :

$$
\begin{aligned}
& R \sim\left(\frac{N}{g_{\Lambda}}\right)^{1 / 3} \xi_{\Lambda} \\
& R \sim N^{1 / 3} \Lambda^{-1 / 6}\left(C a^{3}\right)^{-1 / 8} a
\end{aligned}
$$

in agreement with our previous result $(22 a)$ in the bulk.

The concentration due to the chain itself in the sphere of radius $R$ is

$$
C_{\mathrm{s}} \sim \frac{N}{R^{3}} \sim \Lambda^{1 / 2}\left(C a^{3}\right)^{3 / 8} a^{-3}
$$

so that the number $N_{\mathrm{b}}$ of overlapping chains in this volume is

$$
N_{\mathrm{b}} \sim \Lambda^{-1 / 2}\left(C a^{3}\right)^{5 / 8}
$$

growing from $N_{\mathrm{b}}(\tilde{C}) \sim 1$ to the bulk value when concentration is increased.

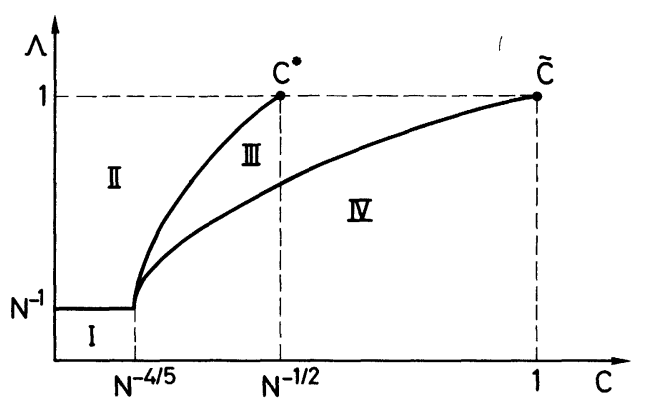

Fig. 4. - The different regimes in a $(\Lambda, C)$ plane, for $d=3$. I : dilute linear $\left(R \sim N^{3 / 5}\right)$; II : dilute branched $\left(R \sim N^{1 / 2} \Lambda^{-1 / 10}\right)$; III : semi dilute, non overlapping $R \sim\left(\frac{N}{C}\right)^{1 / 3}$, see (4.2.a); IV : semi dilute overlapping regime $R \sim N^{1 / 3} \Lambda^{-1 / 6} C^{-1 / 8}$, see (4.2.b).
If we decrease $\Lambda$, when $\Lambda \sim N^{-1}$, we recover the cross-over to the linear chain behaviour. Note that in the process, the non overlapping regime disappears, as $C^{*}$ and $\tilde{C}$ coalesce (see Fig. 4).

Finally, with these results in hand, we may calculate the thickness $\lambda$ of the interfacial layer in the overlapping regime described in the previous section (region 3 of figure 4). The limiting conditions for this interfacial region are that on the border between the non overlapping and overlapping regimes, it is of the order of the radius of the chain

$$
\lambda(\tilde{C}) \sim R .
$$

On the other hand, at $C^{*}$, it is of the order of the linear blob. (We neglect possible logarithmic corrections) :

$$
\lambda\left(C^{*}\right) \sim \xi_{\Lambda} \sim \Lambda^{-3 / 5} a .
$$

Scaling arguments lead then to

$$
\lambda \sim N^{1 / 3}\left(\mathrm{Ca}^{3}\right)^{2 / 3} \Lambda^{-4 / 5} a .
$$

Note that this interface thickness grows in the non overlapping regime when concentration is increased :

$$
\frac{\lambda}{R} \sim \frac{C}{\tilde{C}}
$$

5. Conclusion. - We have studied the conformational properties of randomly branched solutions within a Flory approach. We recovered some results by de Gennes [3] and Isaacson and Lubensky [2] especially about the upper critical dimension above which the classical Zimm-Stockmayer [6] results are valid. The critical dimensions are respectively 8,6 and 4 for a single chain in a good solvent, a theta solvent and the melt. Below these critical dimensions; we gave the scaling laws for the radius of gyration and the pair correlation function. Our result in the melt is different from what is found in reference [2] : we find that the molecules are collapsed, and thus the exponent for the power law dependence of $R$ on the molecular weight $\left(R \sim N^{v} a\right)$ is $d^{-1}$. An interesting point concerns the overlap of the molecules when concentration is increased : if we consider a molecule made of trifunctional units only $(\Lambda=1)$, there is no overlap for $d<4$ which is the case of interest. When bifunctional units are introduced $(\Lambda<1)$, there is a concentration range, $C^{*}<C<\tilde{C}$, where the different molecules do not overlap. If concentration is too high $(C>\widetilde{C})$ however, there is an overlap of the molecules, although it is much lower than for a linear solution in the same conditions (same $C$ and $N$ ). In the limit of purely branched chains $(\Lambda=1), \widetilde{C} \sim 1$ and the second region disappears. On the other hand, for linear chains $(\Lambda N \sim 1) C^{*}$ and $\tilde{C}$ merge and the non overlapping regime does not exist any more. 


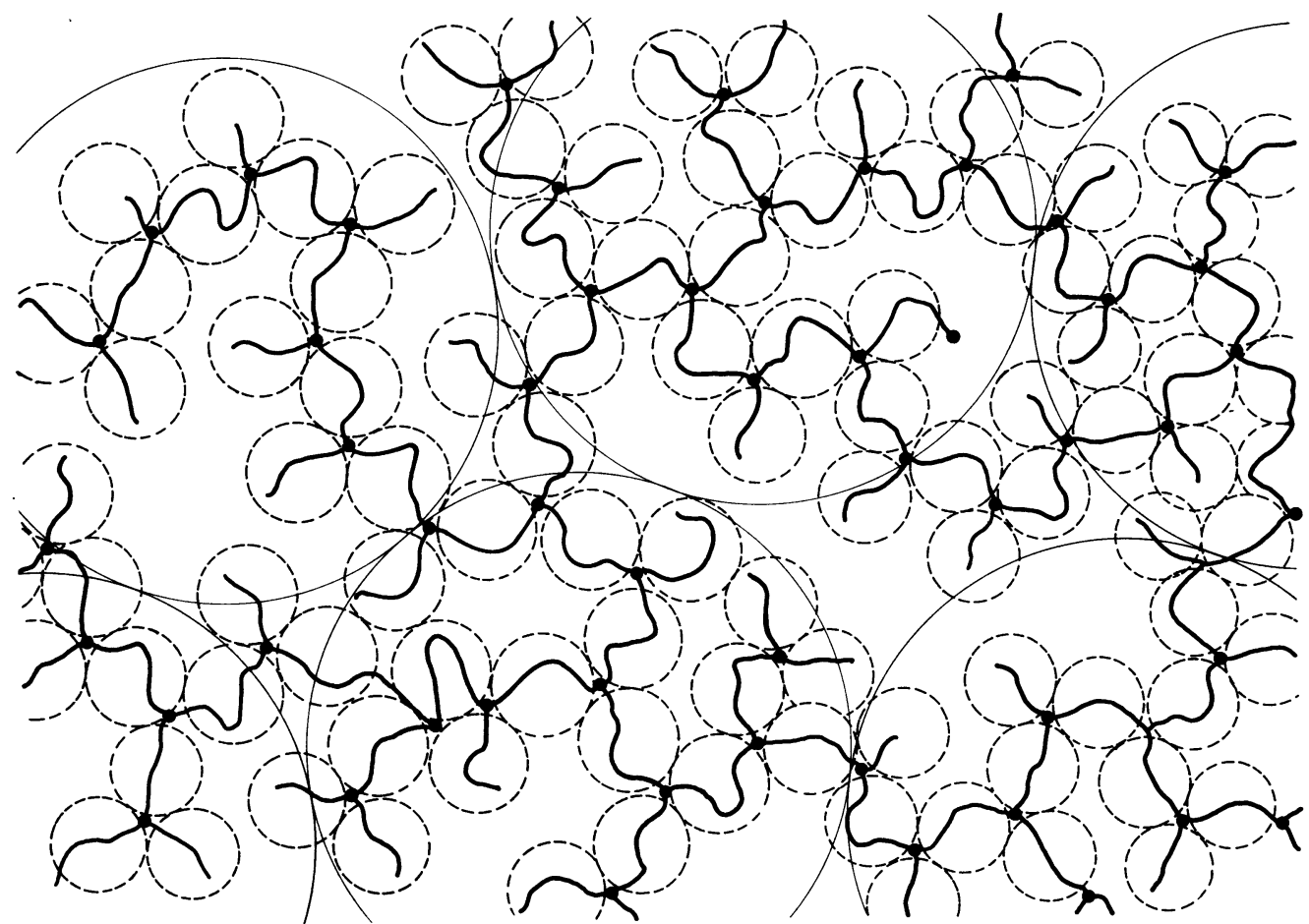

a)

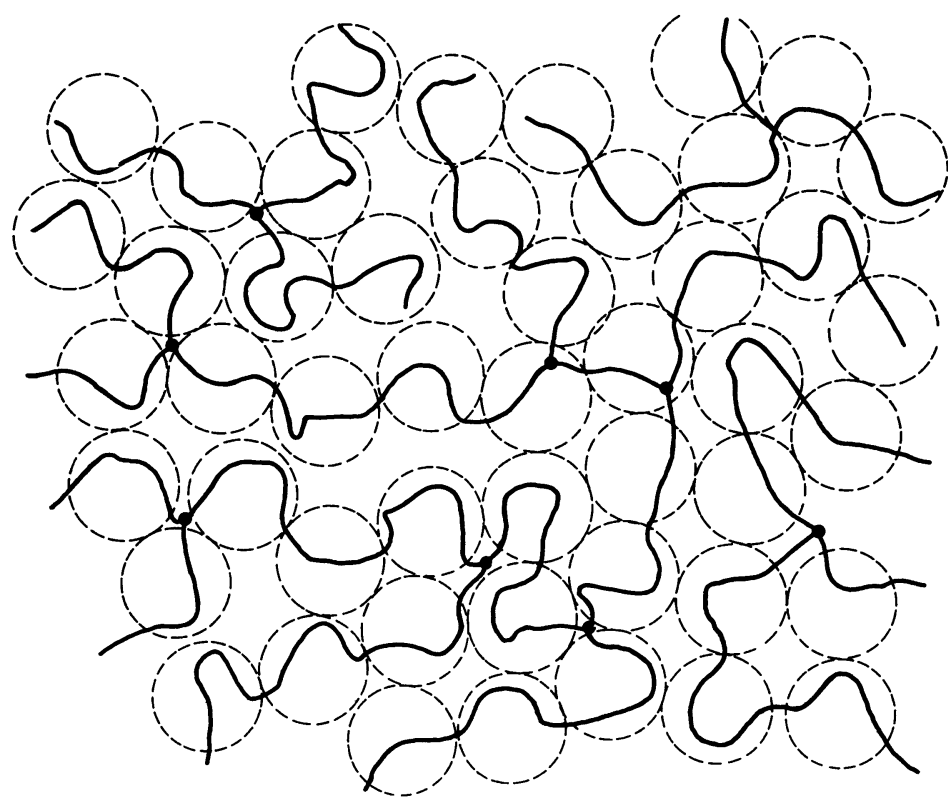

b)

Fig. 5. - Schematic representation of the concentration blobs in the semi dilute ranges III and IV. (a) The structure inside the concentration blob (large circles) is branched and is the same as in figure 1 for a single chain. The size $\xi_{\mathrm{c}}$ of this blob is much larger than that of a linear blob $\xi$. In this regime (III in Fig. 4) there is no overlap of the molecules. (b) For higher concentrations, or smaller $A$, the concentration blob has a linear structure. Its size $\xi_{\mathrm{c}}$ is smaller than the average distance $\zeta$ between 3-functional units. In this regime (IV in Fig. 4) the chains do overlap. The cross-over between $(a)$ and $(b)$ corresponds to line $\tilde{C}$ on figure 4. 
For a single chain in a theta solvent, we find

$$
v=\frac{7}{4(d+1)},
$$

which is a new result as far as we know.

Flory's approach has however at least two shortcomings. First we know that although it gives good numerical results (at least for linear chains in a good solvent), it is not exact [11]. For instance, it can be checked that the $\varepsilon$ expansion $\left(\varepsilon=d_{\mathrm{c}}-d\right)$ of Flory's exponent is in disagreement with Lubensky and Isaacson's results both in a good and in a theta solvent. Moreover, it has been argued that the numerical agreement comes from a compensation between two contributions to the free energy that are not taken into account by Flory's approach, and we do not really know when there is actually a compensation. However we can compare our results with the best estimations, and the result is quite good : in a good solvent for $d=3$, the exact result [17] is $v=1 / 2$. For $d=2$, it is very close to the best computer simulation estimates $[15,21]$. In the melt, the result is consistent with other analytical arguments, at least for $d \leqslant 4$ for the animal problem [16] and should be model independent.

A final shortcoming of Flory's theory - at least as we stated it here - comes from the fact that branched and linear parts are treated on an equal footing : everything is swollen, or collapsed,.... This is true only under specific conditions that were developed above. In the cases when this is not valid, a blob analysis proves to be very helpful to get the results, especially for the $\Lambda$ dependence of the various quantities we studied.

The Flory approach deals only with trees. (We have just added the interactions to the Zimm-Stockmayer model.) If experimentally, it seems possible to generate only trees [10], in most cases one does also generate loops. For dilute solutions, we know [4] that loop formation is not important, but this might not be true for all situations. Among others, branched polymers generated in a theta solvent might have different configurational properties than those made in a good solvent and then cooled down to a theta solvent. For the latter case, the same analysis as in section (4) can be made. We just mention the results. Here again we suppose that linear and multifunctional units have the same theta temperature. We find that for lower concentrations, $C^{*}<C<\tilde{C}$, there is no overlap

$$
\begin{gathered}
C^{*} \sim N^{-5 / 16} \Lambda^{3 / 16} a^{-3} \quad \tilde{C} \sim \Lambda^{1 / 2} a^{-3} \\
R \sim\left(\frac{N}{C}\right)^{1 / 3}
\end{gathered}
$$

with the existence of a linear blob, with size $\xi_{\Lambda} \sim \Lambda^{-1 / 2} a$, and branched blob with size

$$
\xi_{\mathrm{c}} \sim \Lambda^{1 / 5} C^{-7 / 5} .
$$

For higher concentrations $(C>\widetilde{C})$ the concentration blob is also linear [20] :

$$
\begin{aligned}
& \xi_{\mathrm{c}} \sim C^{-1} a^{-2} \\
& \xi_{\Lambda} \sim \Lambda^{-1 / 2}
\end{aligned}
$$

and the molecule is still collapsed : $R \sim N^{1 / 3} \Lambda^{-1 / 6}$. Note that in this regime, the radius does not depend on concentration.

In all our approach, we considered monodisperse samples. The influence of the polydispersity is very interesting to look at. If it is small, we expect our results to hold. However there is one important situation for which our analysis does not apply : if the molecular weight distribution is the one for the gelation problem [9], we expect the percolation exponent to appear. Thus it would be very interesting to introduce the polydispersity in the model and study this cross-over to the percolation problem. For instance, in the gelation problem the molecules do overlap, and it would be very interesting to see how this occurs. Also loops are known to be important in percolation theory and do not seem to be important in the problem studied in the present paper. One would like to understand when and how their importance grows.

Acknowledgments. - The authors are much indebted to J. P. Cotton, P. G. de Gennes, G. Jannink, P. Pincus and D. Stauffer for stimulating discussions and comments.

Appendix A. - In this appendix, we calculate the radius of gyration of a randomly branched polymer within the framework of the Zimm-Stockmayer mean field theory. Rather than following the complicated combinatorial analysis of their original paper we prefer the more direct approach by de Gennes [7]. The basic is then to calculate the propagator of a branched chain, i.e. the probability that a chain which has an end point at the origin has another end point at a distance $r$. It is simple to build a diagrammatic expansion for this propagator $G_{N}(\mathbf{r})$

$$
\left.G\right|_{1} ^{2}=G_{0}^{2}+G_{1}^{3} G^{2}
$$

where $G_{0}$ denotes the propagator for a gaussian chain.

We can then easily write down an equation for the Fourier-Laplace transform of $G_{N}(\mathbf{r})$

$$
\begin{aligned}
& \widetilde{G}(\alpha, q)=\int \mathrm{d} \mathbf{r} \int^{\infty} \mathrm{e}^{i q r} \mathrm{e}^{-\alpha N} G_{N}(\mathbf{r}) \mathrm{d} N \\
& G(\alpha, q)=\widetilde{G}_{0}(\alpha, q)\left[1+\Lambda^{2} \widetilde{G}(\alpha, q=0) \widetilde{G}(\alpha, q)\right]
\end{aligned}
$$


where $\Lambda^{2}$ is the activity of the trifunctional units, and thus the weight of the vertex in (A.0). The propagator $G_{0}$ for a gaussian chains is $[10,11]$

$$
\widetilde{G}_{0}=\frac{1}{\alpha+q^{2} a^{2} / 6} .
$$

Then solving (A.1) gives

$$
\widetilde{G}^{-1}(\alpha, q)=\frac{q^{2} a^{2}}{6}+\frac{1}{2}\left[\alpha+\sqrt{\alpha^{2}-4 \Lambda^{2}}\right] .
$$

Taking the inverse Laplace transform we get

$$
Z_{N}=G_{N}(q=0)=\frac{I_{1}(2 \Lambda N)}{\Lambda N}
$$

where $I_{1}$ is the modified Bessel function of the first kind

$$
G_{N}(q)=2 \sum_{0}^{\infty}(-1)^{n}\left(\frac{q^{2} a^{2}}{3}\right)^{n} \frac{n+1}{(2 \Lambda)^{n+1}} \frac{I_{n+1}(2 \Lambda N)}{N} .
$$

In the high branching limit $\Lambda N \gg 1$ the propagator reduces to

$$
G_{N}(q)=\frac{Z_{N}}{\left(1+\frac{q^{2} a^{2}}{6 \Lambda}\right)^{2}}
$$

which is very similar to the mean field propagator of the field theory by Parisi and Sourlas [17].

In the low branching limit $\Lambda N \ll 1$ we recover the propagator of a linear gaussian chain

$$
G_{N}(q)=\exp \left(-\frac{q^{2} N a^{2}}{6}\right) .
$$

From the partition function $Z_{N}$, one can derive the number $N_{\mathrm{b}}$ of branching points

$$
N_{\mathrm{b}}=\frac{\Lambda N}{Z_{N}} \frac{\partial Z_{N}}{\partial(2 \Lambda N)} .
$$

In the two limits $\Lambda N \gg 1$ and $\Lambda N \ll 1$, equation (A.7) reduces to

$$
N_{\mathrm{b}}=\Lambda N \text {. }
$$

So the limit $\Lambda N \rightarrow 0$ gives back the statistics of linear chains (equation (A.6) is actually the propagator of a gaussian chain) and the limit $\Lambda N \gg 1$ is the case of highly branched trees. The cross-over occurring for $\Lambda N \sim 1$.

The number of end points $N_{\mathrm{e}}$ on the tree is simply related to the number of branching points $N_{\mathrm{b}}$. If the functionality of a branching point is $f$, we have

$$
N_{\mathrm{e}}=N_{\mathrm{b}}(f-2)+2 \text {. }
$$

This means that for highly branched polymers, the number of end points scales like $\Lambda N$ and is very large.

In order to calculate the radius of gyration, we index every monomer by $i(i \in[1, N])$. The position of monomer $i$ is given by a vector $\mathbf{r}_{i}$. The radius of gyration of a polymer is

$$
R_{\mathrm{G}}^{2}=\frac{1}{2 N^{2}} \sum_{i j}\left(\mathbf{r}_{i}-\mathbf{r}_{j}\right)^{2} .
$$

For each pair of monomers $i$ and $j$ let us introduce the linear part of the chain going from $i$ to $j$. We index the monomers along this sequence by $q$ and the vector $\mathbf{r}^{q+1}-\mathbf{r}^{q}$ is denoted by $b_{i j}^{q}$. Its length is always equal to the step length $a$. Because we neglect the excluded volume effects, the chain of $b$ vectors between $i$ and $j$ is a random walk. Thus we can write the average radius of gyration

$$
\left\langle R_{\mathrm{G}}^{2}\right\rangle=\frac{1}{2 N^{2}} \sum_{i j} \sum_{q}\left(b_{i j}^{q}\right)^{2}
$$

where monomer $q$ in the second sum belongs to the sequence $(i j)$.

The problem now is to count the number of configurations in which monomer $q$ belongs to the chain $i j$. In order to do so, following Kramers [19] we cut the chain at monomer $q$. The probability of getting two parts of $n$ and $N-n$ monomers is proportional to $Z_{n} Z_{N-n}$, and in this case, we have $2 n(N-n)$ choices for the pair $i, j$ ( $i$ has to belong to one part of the chain, $j$ to the other). The average radius of gyration is then

$$
\left\langle R_{\mathrm{G}}^{2}\right\rangle=\frac{1}{N} \frac{\sum_{n} n(N-n) Z_{n} Z_{N-n}}{\sum_{n} Z_{n} Z_{N-n}}
$$

which is Kramers' formula.

Inserting the relation for $Z_{n}$ and $Z_{N-n}$ is (A.12) we recover the Zimm-Stockmayer result (Eq. (1)).

Appendix B : Elastic free energy of the chain. We have to calculate the centred correlations of the squared radius of gyration, $\left\langle R_{\mathrm{G}}^{4}\right\rangle-\left\langle R_{\mathrm{G}}^{2}\right\rangle^{2}$. In order to do so, we generalize Kramers' formula (A.12) to this problem $[18,19]$.

For a given configuration

$$
R_{\mathrm{G}}^{4}=\frac{1}{4 N^{4}} \sum_{i j} \sum_{k l} r_{i j}^{2} r_{k l}^{2} .
$$

As previously for the radius gyration, we introduce for each pair $i j$ (or $k l$ ) the linear chain connecting $i$ to $j$ (or $k$ to $l$ ) and the vectors $\mathbf{b}_{i j}^{q}$

$$
\mathbf{r}_{i j}=\sum_{q \in(i, j)} \mathbf{b}_{i j}^{q} .
$$

Thus we have :

$$
R_{\mathrm{G}}^{4}=\frac{1}{4 N^{4}} \sum_{i j} \sum_{k l}\left(\sum_{q \in(i, j)} \mathbf{b}_{i j}^{q}\right)^{2}\left(\sum_{q^{\prime} \in(k, l)} \mathbf{b}_{k l}^{q^{\prime}}\right)^{2} .
$$

Expanding the squares and taking the average, there remains two kinds of non vanishing terms :

(i) terms of the form $\left(b_{i j}^{q}\right)^{2}\left(b_{k l}^{q^{\prime}}\right)^{2}$ with $q$ and $q^{\prime}$ belonging respectively to the sequences $(i, j)$ and $(k, l)$. Summing over these terms gives back $\left\langle R_{\mathrm{G}}^{2}\right\rangle^{2}$. 
(ii) terms of the form $\left(b_{i j}^{q} b_{i j}^{q^{\prime}}\right)^{2}$ where both $q$ and $q^{\prime}$ belong either to $(i j)$ or to $(k l)$. Each of these terms has a contribution $a^{2} / 3$.

Then we can write

$$
\left\langle R_{\mathrm{G}}^{4}\right\rangle-\left\langle R_{\mathrm{G}}^{2}\right\rangle^{2}=\frac{a^{4}}{12 N^{4}} \sum_{i j k l} \sum_{q q^{\prime}} 1
$$

with the above prescription for $q$ and $q^{\prime}$.

$$
\frac{n}{i k} \quad \frac{N-n-P}{j l}
$$

Fig. 2.

In order to calculate the sum, we cut the polymer in 3 parts. The probability of having $n$ monomers in the first part and $p$ in the last is proportional to $Z_{n} Z_{p} Z_{N-n-p}$. The number of choices is $4 n^{2} p^{2}$ (see Fig. 2). The summation over $q$ and $q^{\prime}$ brings in a factor $N(N-n-p)$. So we have

$$
\begin{aligned}
& \left\langle R_{\mathrm{G}}^{4}\right\rangle-\left\langle R_{\mathrm{G}}^{2}\right\rangle^{2}= \\
& =\frac{q^{4}}{3 N^{3}} \frac{\sum_{n, p}(N-n-p) n^{2} p^{2} Z_{n} Z_{p} Z_{N-n-p}}{\sum_{n, p} Z_{n} Z_{p} Z_{N-n-p}} .
\end{aligned}
$$

By taking its Laplace transform, one can calculate the denominator

$$
\sum_{n, p} Z_{n} Z_{p} Z_{N-n-p}=\frac{3}{2 \sqrt{\pi}} \frac{\mathrm{e}^{2 \Lambda N}}{\Lambda^{7 / 2} N^{3 / 2}} .
$$

The numerator is somewhat less direct to calculate, but transforming the sum into an integral, one can perform the calculation analytically

$$
\sum n^{2} p^{2}(N-n-p) Z_{n} Z_{p} Z_{N-n-p}=\frac{N^{5 / 2}}{60 \sqrt{\pi} \Lambda^{9 / 2}} \mathrm{e}^{2 \Lambda N} .
$$

So we end up with

$$
\left\langle R_{\mathrm{G}}^{4}\right\rangle-\left\langle R_{\mathrm{G}}^{2}\right\rangle^{2}=\frac{N a^{4}}{270 \Lambda} .
$$

The important point here is that $\left\langle R_{\mathrm{G}}^{4}\right\rangle-\left\langle R_{\mathrm{G}}^{2}\right\rangle^{2}$ scales both in $N$ and $\Lambda$ in the same way as $\left\langle R_{\mathrm{G}}^{2}\right\rangle^{2}$.

The elastic free energy for a randomly branched polymer is then

$$
\frac{F}{T}=\frac{135}{2} \sqrt{\pi} \frac{\delta R^{2}}{\left(\frac{N}{\Lambda}\right)^{1 / 2} a}=135 \frac{\pi}{8} \frac{\delta R^{2}}{\left\langle R_{\mathrm{G}}^{2}\right\rangle} .
$$

\section{References}

[1] Arvonny, M., Le Monde, Dec. 10 (1980) 13.

[2] IsaAcson, J., Lubensky, T. C., J. Physique Lett. 41 (1980) L-469.

[3] De Gennes, P. G., C. R. Hebd. Séan. Acad. Sci. B 291 (1980) 17.

[4] Isaacson, J., Lubensky, T. C., Phys. Rev. A 20 (1979) 2130. J. Physique 42 (1981) 175.

[5] Family, F., J. Phys. A 13 (1980) L-325.

[6] Zimm, B., Stockmayer, W. H., J. Chem. Phys. 17 (1949) 1301.

[7] De Gennes, P. G., Biopolymers 6 (1968) 715.

[8] Domb, C., J. Phys. A 9 (1976) L-141.

[9] For a description of the percolation problem, the size distribution function and the lattice animals, see the excellent review by Stauffer, D., Phys. Rep. 54 (1979) 1.

[10] Flory, P. J., Principles of Polymer Chemistry, (Cornell Univ. Press) 1953.

[11] De Gennes, P. G., Scaling concepts in Polymer Physics, (Cornell Univ. Press) 1979.
[12] Edwards, S. F., Proc. Philos. Soc. (London) 88 (1966) 265

[13] This is also true for linear melts, where it can be shown by this same method that $v=d^{-1}$ for $d \leqslant 2$.

[14] Daoud, M., Cotton, J. P., Farnoux, B., Jannink, G., Sarma, G., Benoit, H., Duplessix, R., Picot, C. and DE GENNES, P. G., Macromolécules 8 (1975) 804.

[15] Redner, S., J. Phys. A 12 (1979) L-239.

[16] Family, F., Coniglio, A., J. Phys. A 13 (1980) L-403.

[17] Parisi, G., Sourlas, N., Phys. Rev. Lett. 46 (1981) 871.

[18] Dobson, G. R., Gordon, M., J. Chem. Phys. 41 (1964) 2389.

[19] Kramers, H. A., J. Chem. Phys. 14 (1946) 415.

[20] Cotton, J. P., Nierlich, M., Boué, F., Daoud, M., Farnoux, B., Jannink, G., Duplessix, R., Picot, C., J. Chem. Phys. 65 (1976) 1101

[21] Holl, K., unpublished, finds $v=0.53 \pm 0.02$. 